\title{
Computerbasierte Therapien: Eine Ergänzung in der modernen Psychotherapie? - Pro
}

\author{
Computer-Based Therapies: A Supplement in Modern Psychotherapy? - Pro
}

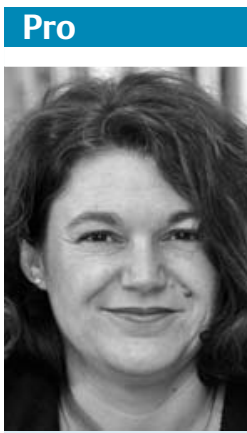

Steffi G. RiedelHeller schen Denken, Fühlen und Handeln zu be-

entsteht, zum anderen mittels Reflektionstechniken und praktischer Übungen. Was vermögen computerbasierte Selbstmanagement- oder Therapieprogramme hier auszurichten? Passt das überhaupt zusammen?

Mit dem Begriff „computerbasierte Interventionen“ sind sowohl Fernbehandlungsansätze über audiovisuelle Medien oder E-Mail gemeint als auch Selbstmanagementprogramme mit und ohne therapeutische Unterstützung. Gibt es therapeutische Unterstützung im Hintergrund, könnte man eine wie auch immer geartete therapeutische Beziehung in Erwägung ziehen. Aber wie sieht es mit den computerbasierten Programmen aus, die ohne therapeutische Unterstützung auskommen? Der folgende Beitrag nimmt gerade diese Programme in den Blick und bezieht sich beispielhaft auf das von der Australian National University entwickelte MoodGYM-Programm (www.moodgymdeutschland.de).

\section{Können Selbstmanagement- programme der Individualität des Menschen gerecht werden?}

In der Tat vermitteln die meisten Selbstmanagementprogramme zunächst allgemeine psychoedukative Informationen und Prinzipien der kognitiven Verhaltenstherapie. Auf dieser Basis werden die Nutzer aber dazu angeleitet, in ihrem Alltag beispielsweise den Zusammenhang zwiobachten, positive Aktivitäten zu planen und festzustellen, wie sich deren Umsetzung auf die eigene Stimmung auswirkt. Die Nutzer werden so in die Lage versetzt, aus allgemeinen Informationen Schlussfolgerungen für ihre individuelle Situation zu ziehen und diese konkret zu verbessern. Sie erwerben also nicht nur theoretisches Wissen, sondern werden durch die Programme ermutigt, ihre sozialen Kontakte zu intensivieren, frühere Hobbys wieder aufzunehmen und diese und weitere positive Aktivitäten Schritt für Schritt umzusetzen.

pressiven Patienten untersucht [7]. Dabei wurde MoodGYM als Selbsthilfeinstrument eingesetzt, das Hausärzte ihren Patienten ergänzend zur regulären Depressionsbehandlung empfehlen konnten. Bei denjenigen, denen zusätzlich MoodGYM empfohlen wurde, besserte sich die depressive Symptomatik nach 6 Wochen und nach 6 Monaten signifikant stärker als bei denjenigen, die ausschließlich die gewöhnliche hausärztliche Versorgung erhielten. Diese Ergebnisse stehen in Einklang mit internationalen Studien, die ähnliche Resultate erbrachten (u.a. [8, 9]). Die Ausnahme macht eine aktuelle britische Studie, die Selbstmanagementprogramme (MoodGYM und Beating the Blues) bei hausärztlich behandelten depressiven Patienten einsetzte, und keine Vorteile zeigen konnte [10]. Das mag auch daran liegen, dass MoodGYM dort seit Jahren frei verfügbar ist. Daher nutzte auch die Kontrollgruppe zu fast 20\% MoodGYM oder ein anderes Selbstmanagementprogramm. Zudem waren die Patienten in der britischen Studie schwerer krank und wurden durch technisches Studienpersonal außerhalb der eigentlichen Behandlungsbeziehung zum Hausarzt ermutigt, das Programm zu nutzen.

\section{Selbstmanagementprogramme sind wirksam}

Zahlreiche Metaanalysen zeigen die Wirksamkeit von Selbstmanagementprogrammen in der Behandlung von Substanzgebrauchsstörungen, Angststörungen, posttraumatischen Belastungsstörungen und Essstörungen [1]. Auch bei subklinischen und manifesten Depressionen ist die Wirksamkeit von Selbstmanagementprogrammen gut belegt (u.a. [2-5]). Das britische National Institute for Health and Care Excellence empfiehlt diese Selbstmanagementprogramme deshalb als psychosoziale Interventionen niedriger Intensität im Rahmen eines gestuften Behandlungsprogrammes bei Depression [6]. Wir haben die Wirksamkeit der deutschen MoodGYM-Version in einer cluster-randomisierten Studie mit 647 de-
Vier gute Gründe, die Chancen von Selbstmanagementprogrammen zu nutzen

1. Enormer Bedarf: Psychische Störungen sind häufig. Es spricht viel dafür, alle Möglichkeiten auszuschöpfen, um diesen Menschen Unterstützung und Zugang zu psychoedukativem Wissen zu geben. Dieses Wissen muss einfach aufbereitet sein, damit Nutzer mit unterschiedlichem Bildungsniveau davon profitieren können.

2. Möglicher Einstieg in eine fachspezifische Behandlung: Selbstmanagementprogramme können als Einstieg in eine Psychotherapie dienen - gerade für Menschen, die aus bestimmten Erwägungen, z.B. aus Angst vor Stigmatisierung oder Überfor- 
derung, (noch) keine psychotherapeutische Hilfe suchen wollen.

3. Besondere Umstände: Auch in Deutschland kann es, gerade in ländlichen Gegenden, schwer sein, zeit- und wohnortnah psychotherapeutische Unterstützung zu erhalten. Zudem können Mobilitätseinschränkungen oder andere Umstände, den Zugang zu Beratung oder fachspezifischer Behandlung erschweren. Selbstmanagementprogramme können dabei helfen, solche Barrieren zu überwinden oder Wartezeiten zu überbrücken.

4. Empowerment fördern: Das Internet hat die Informationsasymmetrie zwischen Experten und Patienten deutlich verringert. Das ist gut so. Damit ermöglichen sie es den Patienten einerseits, sich intensiver auf Face-to- Face-Therapien vorzubereiten und erlauben andererseits eine gezielte selbstständige Vertiefung der in der Therapie vermittelten Inhalte.

\section{Werden Psychiater überflüssig?}

Wir sehen ein enormes Potenzial von Selbstmanagementprogrammen als „addon" zur regulären Versorgung - auch in psychiatrischen Ambulanzen, Kliniken und psychotherapeutischen Praxen. Behandler berichteten, dass sie ihren Patienten solche Programme empfehlen, weil damit auch basale psychoedukative Kenntnisse vermittelt werden und sie dann in der Therapie fokussierter am Kernproblem arbeiten können. Auch sehen wir keinen Anhaltspunkt, dass Therapeuten überflüssig werden, ganz im Gegenteil. Menschen können für das Anliegen von Therapie sensibilisiert werden und möglicherweise eher den Weg in eine Behandlung finden. In den Informa- tionsmaterialien für Ärzte und Patienten und den Nutzungsbedingungen zu MoodGYM wird klargestellt, dass das Programm bei manifesten Depressionen nur unterstützend zur ärztlichen Behandlung eingesetzt werden soll und keine fachgerechte Diagnose oder Behandlung durch einen Arzt oder Psychotherapeuten ersetzt. Die Entwicklung der deutschen MoodGYM-Version wurde von der AOK initiiert und gefördert. Das Programm steht nicht nur AOK-Versicherten zur Verfügung, sondern kann von allen frei und kostenlos genutzt werden. Damit können Ärzte und Psychotherapeuten MoodGYM allen Patienten empfehlen, für die es aus medizinischer Sicht geeignet ist.

\section{Literatur}

1 Klein JP, Gerlinger G, Knaevelsrud C et al. Internetbasierte Interventionen in der Behandlung psychischer Störungen. Überblick, Qualitätskriterien, Perspektiven. Nervenarzt; DOI: 10.1007/s00115-016-0217-7

2 Richards D, Richardson T. Computerbased psychological treatments for depression: a systematic review and meta-analysis. Clin Psychol Rev 2012; 32: 329-342

3 Cuijpers $P$, Donker T, Johansson $R$ et al. Selfguided psychological treatment for depressive symptoms: a meta-analysis. PLoS ONE; DOI: 10.1371/journal.pone.0021274

4 Foroushani PS, Schneider J, Assareh N. Metareview of the effectiveness of computerised CBT in treating depression. BMC Psychiatry 2011; 11: 131

5 Sikorski C, Luppa M, Kersting A et al. Computer-aided Cognitive Behavioural Therapy for depression: a systematic review of the literature. Psychiat Prax; DOI: 10.1055/s-00311277921

6 National Institut for Health and Care Excellence NICE. Guideline: Depression in adults: recognition and management. (01.10.2009). Im Internet: www.nice.org.uk/guidance/ cg90/chapter/1 guidance? unlid=136985012 01599233454\#step-1-recognition-assess ment-and-initialmanagement

(Stand 09.08.2016)

7 Riedel-Heller SG, Pabst A, Löbner $M$ et al. MoodGYM as computerised cognitive behaviour therapy (CCBT) embedded in primary care: patients with mild to moderate depression benefit. Results of a large scale cluster randomised controlled trial (AKTIVTrial). 2016: (submitted)

8 Proudfoot J, Ryden C, Everitt B et al. Clinical efficacy of computerised cognitive-behavioural therapy for anxiety and depression in primary care: randomised controlled trial. Br J Psychiatry 2004; 185: 46-54

9 Hickie IB, Davenport TA, Luscombe GM et al. Practitioner supported delivery of internetbased cognitive behaviour therapy: evaluation of the feasibility of conducting a cluster randomised trial. Med J Australia 2010; 192 : S31-S35

10 Gilbody S, Littlewood E, Hewitt C et al. Computerised cognitive behaviour therapy (cCBT) as treatment for depression in primary care (REEACT trial): large scale pragmatic randomised controlled trial. BMJ 2015; 351: h5627

Sie haben eine eigene Meinung zu diesem

Thema? Dann schreiben Sie uns an:

psychiat-praxis@thieme.de!

\section{Korrespondenzadresse}

\section{Prof. Dr. Steffi G. Riedel-Heller, MPH}

Universitätsklinikum Leipzig, Institut für Sozialmedizin, Arbeitsmedizin und Public Health (ISAP)

Philipp-Rosenthal-Straße 55

04103 Leipzig

steffi.riedel-heller@medizin.uni-leipzig.de

\section{Bibliografie}

Dol http://dx.doi.org/

10.1055/s-0042-115320

Psychiat Prax 2017; 44: 10-11

(c) Georg Thieme Verlag KG

Stuttgart - New York

ISSN 0303-4259 